\title{
Estimativa de biomassa e carbono total para árvores de caixeta no Paraná
}

\author{
Lara Clímaco de Melo ${ }^{1}$, Carlos Roberto Sanquetta ${ }^{1}$, Ana Paula Dalla Corte ${ }^{1}$, Ângela Maria Klein Hentz
}

${ }^{1}$ Universidade Federal do Paraná, Departamento de Engenharia Florestal , Av. Pref. Lothário Meissner, 900, Jardim Botânico - Campus III, 80210-170, Curitiba, PR, Brasil

"Autor correspondente:

laracmelo@gmail.com

Termos para indexação:

Modelagem

Mata Atlântica

Fixação de carbono

Tabebuia cassinoides

Index terms:

Modeling

Atlantic Forest

Carbon fixation

Tabebuia cassinoides

Histórico do artigo:

Recebido em 16/09/2013

Aprovado em 18/03/2014

Publicado em 04/04/2014

doi: 10.4336/2014.pfb.34.77.592
Resumo - O objetivo deste trabalho foi realizar o ajuste de modelos para estimar biomassa e carbono total de indivíduos de caixeta (Tabebuia cassinoides (Lam.) DC.), localizado em Guaratuba, litoral Paranaense, Brasil. Foram testados 13 modelos, e a escolha do melhor baseou-se nos indicadores estatísticos $\mathrm{R}_{\mathrm{aj}}^{2}, \mathrm{~S}_{\mathrm{yx}}(\%)$ e análise gráfica dos resíduos. Realizou-se ainda, como critério complementar de escolha, a avaliação das condicionantes de regressão dos resíduos dos melhores modelos. Não foram feitos ajustes de biomassa e carbono por compartimentos devido à baixa correlação entre as variáveis dependentes (biomassa e carbono) e independentes, diâmetro à altura do peito (dap) e altura total (h). Para a biomassa total, o modelo $\mathrm{Y}=\beta 0+\beta 1 *$ dap se mostrou superior aos demais, apresentando um $\mathrm{R}^{2}$ aj de 0,96 e $\mathrm{S}_{\mathrm{yx}}(\%)$ de 7,94. Para carbono, melhor ajuste foi obtido pelo modelo $\left(Y=\beta 0+\beta 1 *\right.$ dap $+\beta 2 *$ dap $^{2}+\beta 3 *$ dap $^{3}+\beta 4 *$ dap $^{4}$, com valores de $\mathrm{R}_{\text {aj }}^{2} 0,97$ e $\mathrm{S}_{\mathrm{yx}}(\%) 8,09$. Constatou-se a baixa variação dos resíduos para ambos os modelos. A variável altura total, utilizada de forma isolada, revelou-se inadequada para explicar as variáveis biomassa total e carbono total.

\section{Estimation of yotal biomass and carbon for caixeta trees in Parana State, Brazil}

\begin{abstract}
The objective of this work was to adjust models to estimate biomass and total carbon of individuals of Caixeta (Tabebuia cassinoides (Lam.) DC.), located in Guaratuba, coast of Parana State, Brazil. Thirteen models were tested, and the choice of the best was based on the statistical indicators $\mathrm{R}_{\mathrm{aj}}^{2}, \mathrm{~S}_{\mathrm{yx}}(\%)$ and graphical analysis of residuals. It was also an additional choice criteria, the evaluation of residual from the constrained regression for the best models. No adjustments were made for biomass and carbon by compartments due to the low correlation between the dependent variables (biomass and carbon) and independent, diameter at breast height (dbh) and total height (h). For total biomass, the model $\mathrm{Y}=\beta 0+\beta 1 * \mathrm{dbh}$ presented better results, with $\mathrm{R}^{2}{ }_{\text {aj }}=0.96$ and $\mathrm{S}_{\mathrm{yx}}(\%)=7.94$. For carbon, the best adjustment was given by model $\mathrm{Y}=\beta 0+\beta 1 * \mathrm{dbh}+\beta 2 * \mathrm{dbh}^{2}+\beta 3 * \mathrm{dbh}^{3}+\beta 4 * \mathrm{dbh}^{4}$, with $\mathrm{R}_{\mathrm{aj}}^{2}=0.97$ and $\mathrm{S}_{\mathrm{yx}}(\%)=8.09$. It was noticed the low variation of residues for both models. The variable total height, used isolated has proved to be inadequate to explain the variables total biomass and total carbon.
\end{abstract}




\section{Introdução}

O aumento da concentração de gases de efeito estufa (GEE) na atmosfera terrestre, principalmente de $\mathrm{CO}_{2}$, como resultado das atividades antrópicas, tem implicado na intensificação do efeito estufa natural e, em consequência, nas mudanças climáticas globais (Ribeiro et al., 2010).

Tendo como foco os efeitos do aquecimento global, o Protocolo de Quioto instituiu a redução de GEE por meio de mecanismos de flexibilização, dentre eles o mecanismo de desenvolvimento limpo (MDL), que tem como objetivo ajudar os países do Anexo I do Protocolo de Quioto a alcançarem metas de redução de emissões. Segundo Martins (2011), os projetos de MDL que têm recebido mais destaque atualmente são os de florestamento e reflorestamento.

Mundialmente, há um consenso acerca do importante papel das florestas no processo das mudanças climáticas ao atuar como sumidouros, onde as mesmas absorvem $\mathrm{CO}_{2}$ da atmosfera e armazenam carbono em sua biomassa, constituindo amplos reservatórios desse elemento (Silveira et al., 2008). Um dos procedimentos utilizados para conhecer o estoque de carbono das florestas é através da determinação da biomassa e do desenvolvimento de modelos alométricos para sua estimativa (Mognon et al., 2013).

Silveira (2010) afirma que os métodos atuais de estimações de biomassa em florestas têm como base dados de inventário florestal, onde fatores e equações transformam dados de diâmetro e altura nessas estimativas. Assim, a metodologia de cálculo indireto consiste na correlação da variável pretendida com variáveis de fácil obtenção, evitando a necessidade de destruição das árvores.

Uma das dificuldades para aceitar a proposta florestal no MDL são os entraves relacionados com as metodologias de quantificação da biomassa e estoques de carbono nas diferentes formações florestais (Poppe, 2008). Porém, Miranda et al. (2011) afirmam que o interesse em estudos sobre estoque de biomassa e carbono nos diferentes biomas do Brasil é crescente. No Estado do Paraná, a caixeta (Tabebuia cassinoides (Lam.) DC.) possui relativa importância socioeconômica, devido à facilidade de exploração e sistema de rebrota, sendo útil na confecção de lápis de alta qualidade, instrumentos musicais, artesanatos, entre outros (Galvão et al., 2002).

Diante da possibilidade do uso das florestas como mitigadoras de problemas ambientais, objetiva-se com esse estudo ajustar modelos para estimar o estoque da biomassa e carbono total em povoamento de Tabebuia cassinoides (Lam.) DC., visando a utilização dessas estimativas em projetos que utilizem o potencial de fixação de biomassa e carbono.

\section{Material e métodos}

Os dados coletados para o presente estudo são provenientes de uma área particular (Fazenda Estrela) com predomínio de Tabebuia cassinoides, pertencente à família Bignoniaceae, classificada como formação pioneira de influência flúvio-lacustre pertencente à Floresta Ombrófila Densa de Terras Baixas, explorada para fabricação de lápis, confecção de cerca e artesanato pelos proprietários e comunidade local. O uso da estaquia propiciou uma regeneração abundante da caixeta, configurando uma fitofisionomia de floresta natural. O caixetal em questão tem idade aproximada de 30 anos e localiza-se no Município de Guaratuba, região litoral do Paraná (Figura 1).

Foram avaliados 20 indivíduos de caixeta utilizando o método destrutivo. A escolha das amostras foi realizada considerando a distribuição dos indivíduos de acordo com a média diamétrica da população, conforme demonstrado na Tabela 1.

Tabela 1. Distribuição diamétrica e altimétrica dos indivíduos de T. cassinoides.

\begin{tabular}{cc}
\hline Classes de diâmetro (cm) & Frequência \\
\hline $7-9,24$ & 8 \\
$9,25-11,4$ & 9 \\
$11,50-13,74$ & 2 \\
$13,75-16$ & 1 \\
\hline Classes de altura (m) & \\
\hline $8-9,65$ & 5 \\
$9,66-11,31$ & 11 \\
$11,32-12,98$ & 4 \\
\hline
\end{tabular}

As variáveis mensuradas foram circunferência à altura do peito (cap), altura total (h) e altura do fuste (hf), transformando o cap em dap (diâmetro à altura do peito). Procedeu-se então o abatimento e pesagem das árvores, considerando o método de simples separação (Sanquetta et al., 2004), onde cada indivíduo teve seu peso verde determinado por compartimento (folhas, galhos, fuste sem casca, casca e raízes) em campo por meio de balança mecânica. 


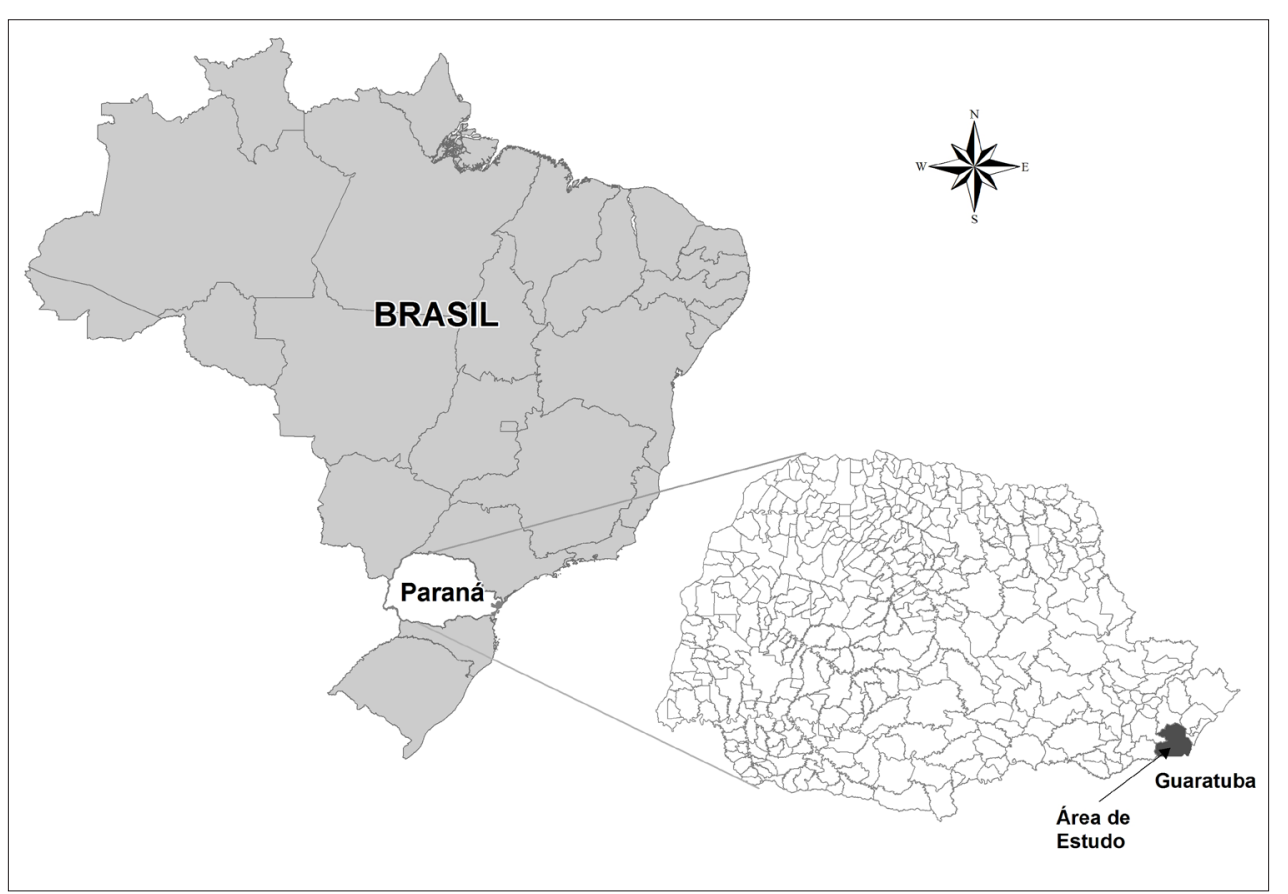

Figura 1. Localização da área de estudo, Guaratuba, Paraná, Brasil.

Para a coleta das raízes, definiu-se a área útil da árvore na floresta, considerando espaçamento do povoamento de $2 \mathrm{~m}$ $\mathrm{x} 2 \mathrm{~m}$, abrindo-se uma trincheira de $50 \mathrm{~cm}$ de profundidade em volta de cada indivíduo selecionado, sendo efetuada a retirada das raízes com diâmetro igual ou superior a $1 \mathrm{~cm}$. Procedeu-se então a limpeza e pesagem dessas raízes.

De posse dos dados de biomassa verde, retiraram-se amostras de 300 e $500 \mathrm{~g}$ de cada compartimento, sendo as mesmas alocadas em sacos de papel, identificadas e levadas do campo em imediato por transporte automotivo. No laboratório, as amostras foram descascadas e seus compartimentos direcionados para secagem em estufa (aproximadamente $70^{\circ} \mathrm{C}$ ) até atingir peso seco constante. Foi realizada moagem das amostras secas visando à obtenção dos teores de carbono, através do analisador de carbono Leco C-144. Neste, a amostra de material sólido é levada à combustão total, e um sensor infravermelho acoplado detecta a quantidade de dióxido carbono $\left(\mathrm{CO}_{2}\right)$ gerado pela combustão, que é relacionado automaticamente com a quantidade de carbono existente na amostra.

Avaliou-se, ainda, a correlação das variáveis utilizadas e, por este resultado, não foram realizados ajustes de biomassa e carbono por compartimentos, tendo em vista a baixa correlação entre as variáveis dependentes (biomassa e carbono) e independentes (dap e h) consideradas. Deste modo, o somatório da biomassa seca de todos os compartimentos mensurados constituiu a biomassa seca total e os teores de carbono por compartimento, multiplicados por suas respectivas biomassas secas também foram somados de modo a se obter o peso seco de carbono total.

A fim de conhecer o comportamento dos dados utilizados no ajuste, realizou-se análise da normalidade, considerando os principais testes, bem como, a estatística descritiva das variáveis, de modo a descrever e informar sobre o conjunto de dados em questão. Assim, foram testados 13 modelos (Tabela 2) descritos na literatura, com vistas a uma estimativa confiável acerca do estoque de biomassa e carbono total da caixeta.

$\mathrm{Na}$ avaliação do desempenho e seleção do melhor modelo para as estimativas, foram considerados os indicadores de ajustes: coeficiente de determinação ajustado $\left(\mathrm{R}_{\text {aj }}^{2}\right)$, erro padrão da estimativa $\left(\mathrm{S}_{\mathrm{yx}} \%\right)$ e análise gráfica dos resíduos. Nos modelos em que a variável dependente sofreu transformação logarítmica, foi aplicado o fator de correção de Meyer para corrigir a discrepância logarítmica. $O$ erro padrão também foi corrigido na escala original, para possibilitar a comparação com os modelos aritméticos.

Os resíduos dos melhores modelos foram avaliados quanto às condicionantes de normalidade (Shapiro-Wilk), homocedasticidade da variância (White) e independência dos resíduos pela estatística de Durbin-Watson (Schneider et al., 2009) utilizando o processo Forward. 
Tabela 2. Modelos testados para estimativa de biomassa e carbono total em T. cassinoides.

\begin{tabular}{|c|c|}
\hline Número & Modelo \\
\hline 1 & $\mathrm{Y}=\beta 0+\beta 1 \mathrm{~h}$ \\
\hline 2 & $Y=\beta 0+\beta 1$ dap \\
\hline 3 & $\mathrm{Y}=\beta 0+\beta 1 \mathrm{dap}^{2 * h}$ \\
\hline 4 & $\mathrm{Y}=\beta 0+\beta 1$ dap $+\beta 2 \mathrm{~h}$ \\
\hline 5 & $\operatorname{Ln} Y=\beta 0+\beta 1 \ln$ dap \\
\hline 6 & $\operatorname{Ln} Y=\beta 0+\beta 1 \ln \operatorname{dap}^{2 * h}$ \\
\hline 7 & $Y=\beta 0+\left(\beta 1 \text { dap }^{0.5}\right)^{*} \ln$ dap \\
\hline 8 & $\mathrm{Y}=\beta 0+\beta 1 *$ dap $+\beta 2 * \operatorname{dap}^{2}+\beta 3\left(\right.$ dap $\left.^{2} \mathrm{~h}\right)$ \\
\hline 9 & $\mathrm{Y}=\beta 0+\beta 1 *$ dap $+\beta 2\left(\right.$ dap $\left.^{2} \mathrm{~h}\right)$ \\
\hline 10 & $\operatorname{Ln} \mathrm{Y}=\beta 0+\beta 1 * \ln$ dap $+\beta 2 * \ln \mathrm{h}$ \\
\hline 11 & $\operatorname{Ln} Y=\beta 0+\beta 1 * \ln \operatorname{dap}^{3}$ \\
\hline 12 & $\mathrm{Y}=\beta 0+\beta 1 *$ dap $+\beta 2 *$ dap $^{2}+\beta 3 *$ dap $^{3}+\beta 4^{*}$ dap $^{4}$ \\
\hline 13 & $\mathrm{Y}=\beta 0+\beta 1 * \mathrm{dap}^{3}$ \\
\hline
\end{tabular}

$\mathrm{Y}=$ biomassa seca total ou carbono total; $\mathrm{Ln}=$ logaritmo neperiano; $\beta_{\mathrm{n}}=$ coeficientes da regressão; dap = diâmetro à altura do peito; $\mathrm{h}=$ altura total.

\section{Resultados e discussão}

Na avaliação das variáveis dependentes quanto à sua normalidade, observou-se distribuição normal para todos os principais testes da literatura considerados (Tabela 3).

Para a estatística descritiva, observou-se que os indivíduos apresentam diâmetro médio de $10,27 \mathrm{~cm}$, variando de 7,59 até 15,91. Quanto aos valores de biomassa e carbono total, obtidos a partir da soma de todos os compartimentos, estes apresentam média de 25,03 e 11,61 kg, com desvios-padrão de 9,83 e 5,04, respectivamente (Tabela 4).

Os resultados descritos estão dentro do esperado para o estágio de desenvolvimento, com indivíduos em regeneração. Batista et al. (2004) encontraram diâmetro e altura médios de $18,8 \mathrm{~cm}$ e $12 \mathrm{~m}$ para indivíduos de caixetas em ambientes naturais nos estados de São Paulo e Rio de Janeiro em estágios médios a avançados. Observou-se ainda a baixa amplitude dos dados das variáveis independentes quando comparado as variáveis dependentes, em especial a biomassa.

Os resultados de alocação média da biomassa demonstraram maior representação do fuste, com 38\% do total (Figura 2A). Essa mesma relação foi observada para o carbono, com valor de $33 \%$ para o fuste (Figura 2B). Em seguida, os maiores percentuais foram dados pelos compartimentos galho, casca, raízes e folhas. Esse resultado foi descrito por Schneider et al. (2004), onde mencionam que geralmente a distribuição aérea da biomassa total se dá na ordem de: lenho $>$ galhos $>$ casca $>$ raízes $>$ folhas.

Tabela 3. Testes de normalidade dos valores de biomassa e carbono total para indivíduos de caixeta no Paraná.

\begin{tabular}{lrrrr}
\hline \multirow{1}{*}{ Testes } & Valor & $\begin{array}{c}\text { Valor } \\
\text { crítico }\end{array}$ & p-valor & Normal \\
\cline { 2 - 5 } & \multicolumn{4}{c}{ Biomassa } \\
\hline $\begin{array}{l}\text { Kolmogorov-Smirnov } \\
\text { (D) }\end{array}$ & 0,17191 & 0,19238 & $\mathrm{p}>0,10$ & Sim \\
$\begin{array}{l}\text { Cramér-von Mises } \\
\text { (W2) }\end{array}$ & 0,10480 & 0,12293 & $\mathrm{p}>0,05$ & Sim \\
Anderson-Darling (A2) & 0,65874 & 0,69187 & $\mathrm{p}>0,05$ & Sim \\
Kuiper (V) & 0,28813 & 0,31644 & $\mathrm{p}>0,10$ & Sim \\
Watson (U2) & 0,08631 & 0,11317 & $\mathrm{p}>0,10$ & Sim \\
Lilliefors (D) & 0,17191 & 0,19000 & $\mathrm{p}>0,10$ & Sim \\
\hline & & Carbono & \\
\hline $\begin{array}{l}\text { Kolmogorov-Smirnov } \\
\text { (D) }\end{array}$ & 0,15436 & 0,19238 & $\mathrm{p}>0,15$ & Sim \\
$\begin{array}{l}\text { Cramér-von Mises } \\
\text { (W2) }\end{array}$ & 0,09840 & 0,12293 & $\mathrm{p}>0,10$ & Sim \\
$\begin{array}{l}\text { Anderson-Darling (A2) } \\
\text { Kuiper (V) }\end{array}$ & 0,60568 & 0,69187 & $\mathrm{p}>0,05$ & Sim \\
Watson (U2) & 0,26889 & 0,31644 & $\mathrm{p}>0,15$ & Sim \\
Lilliefors (D) & 0,08282 & 0,11317 & $\mathrm{p}>0,15$ & Sim \\
\hline & 0,15436 & 0,19000 & $\mathrm{p}>0,15$ & Sim \\
\hline
\end{tabular}

Tabela 4. Estatística descritiva das variáveis dependentes e independentes usadas no ajuste de modelos para estimativa de biomassa e carbono de caixeta.

\begin{tabular}{lccccc}
\hline \multicolumn{1}{c}{ Compartimento } & Média & Mínimo & Máximo & Desvio padrão & $\mathbf{N}^{\mathbf{c}}$ \\
\hline DAP $(\mathrm{cm})$ & 10,270 & 7,958 & 15,915 & 1,942 & 20 \\
HT $(\mathrm{m})$ & 10,403 & 8,120 & 12,400 & 1,305 & 20 \\
Carbono total $(\mathrm{Kg})$ & 11,613 & 5,480 & 23,949 & 5,047 & 20 \\
Biomassa $(\mathrm{Kg})$ & 25,033 & 13,304 & 52,098 & 9,833 & 20 \\
\hline
\end{tabular}


(A)

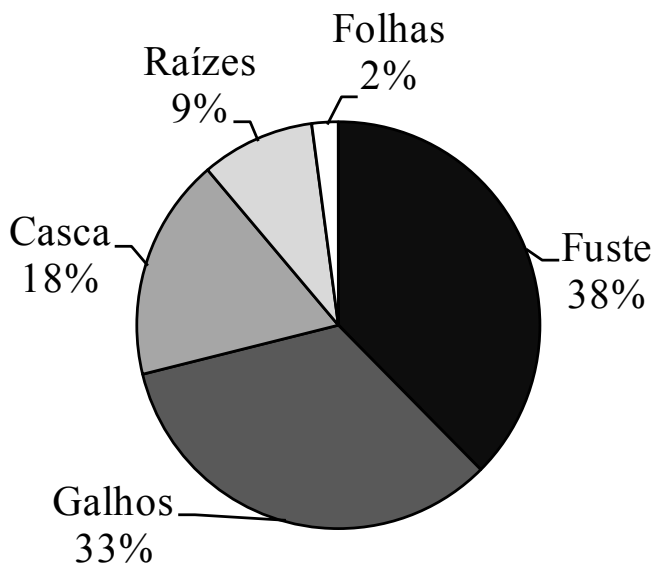

B)

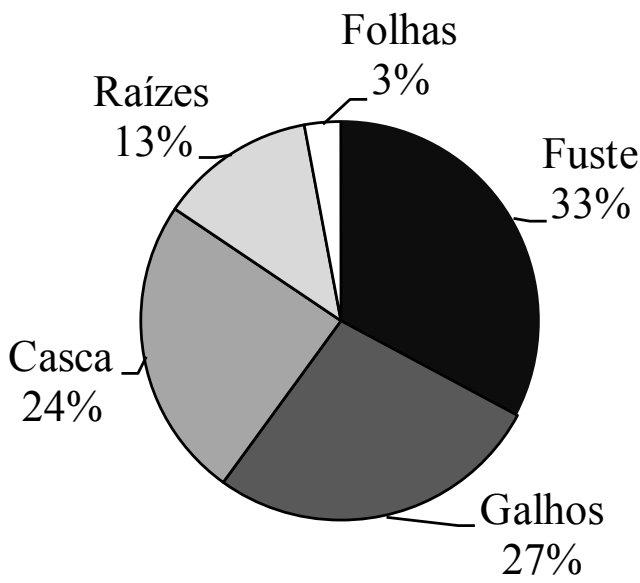

Figura 2. Distribuição dos percentuais de biomassa (A) e carbono (B) nos compartimentos das árvores de T. cassinoides.

Em relação ao ajuste dos modelos para predição de biomassa e carbono nos indivíduos de $T$. cassinoides, todos os modelos apresentaram bons índices de precisão, com exceção do modelo 1 , que tinha como única variável independente a altura. Justifica-se esse desempenho inferior pela baixa correlação da altura com a biomassa e carbono. Os modelos aritméticos apresentaram melhores ajustes em relação aos logarítmicos, comportamento similar ao observado por Silveira (2009) ao estimarem a biomassa de indivíduos de diferentes espécies nativas da Floresta Ombrófila Densa em Santa Catarina.

O melhor $\mathrm{R}_{\text {aj }}^{2}$ para estimativa da biomassa total foi encontrado no modelo 2, representado também pelo menor erro $\left(\mathrm{S}_{\mathrm{yx}} \%=7,94\right)$. Entretanto, os modelos 8,9 e 12, obtiveram valores semelhantes a estes, podendo ser considerados apropriados para estimar a biomassa total, conforme demonstrado na Tabela 5. É importante mencionar que, entre esses modelos, cujos indicadores de ajustes apresentaram bom desempenho, os modelos 2 e 12 utilizam apenas a variável dap para ajuste, de fácil obtenção, devendo ser priorizadas em função dos outros modelos compostos por mais variáveis.

Esses resultados corroboram com os encontrados por Miranda et al. (2011), que observaram valores de 0,96 e 29,77 para $\mathrm{R}_{\text {aj }}^{2}$ e $\mathrm{S}_{\mathrm{yx}}(\%)$ respectivamente, para espécies nativas de rápido crescimento, característica da caixeta, testando modelos para biomassa total e carbono em área de restauração. O menor erro obtido pelo presente estudo pode ser justificado pela homogeneidade dos dados em relação à uma diversidade de espécies, tendo em vista que diferentes tipos florestais possuem diferentes distribuições de biomassa, inferindo-se cautela com o uso de equações que estimaram biomassa e carbono em condições divergentes com a qual está sendo proposta (Koehler et al., 2005).

A distribuição dos resíduos dos modelos selecionados pelos indicadores estatísticos mencionados comprovou a eficácia da estimativa, onde não foram observadas tendências ao longo da linha de estimativa da biomassa total, demonstrado pela Figura 3

Em complementaridade, os resíduos destes modelos foram avaliados quanto às condicionantes da regressão, através do teste de White - W (homocedasticidade), Shapiro-Wilk - SW (normalidade) e Durbin-Watson DW (independência), conforme Tabela 6.

Observa-se que as equações 2, 8 e 12 atendem às três condicionantes testadas, enquanto que a equação 9 atende somente às condições de homocedasticidade e normalidade, porém é significativo a $5 \%$ de probabilidade para o teste de independência, rejeitando a hipótese de que os resíduos sejam independentes. Desta forma, indica-se a preferência aos modelos que atenderam a todas as condições, sendo eles os modelos 2, 8 e 12 . Em análise a todas as estatísticas de ajuste, o modelo 2 teve desempenho superior.

Lacerda et al. (2009), testou diferentes modelos para estimar biomassa e carbono em espécies nativas da Mata Atlântica, e concluiu que o modelo que proporcionou melhor ajuste aos dados foi o que utiliza apenas o dap como variável independente, tanto para biomassa total quanto para os compartimentos fuste e galho, bem como ao mensurar quantidade de carbono das árvores, realidade que corrobora com o observado neste trabalho. 
Tabela 5. Coeficientes e indicadores estatísticos dos modelos para estimativa de biomassa total para T. cassinoides.

\begin{tabular}{crcccccc}
\hline Modelo & $\boldsymbol{\beta}_{\mathbf{0}}$ & $\boldsymbol{\beta}_{\mathbf{1}}$ & $\boldsymbol{\beta}_{\mathbf{2}}$ & $\boldsymbol{\beta}_{\mathbf{3}}$ & $\boldsymbol{\beta}_{\mathbf{4}}$ & $\mathbf{R}_{\text {aj. }}^{\mathbf{2}}$ & $\mathbf{S}_{\mathbf{y x}}(\mathbf{\%})$ \\
\hline 1 & $-13,398$ & 3,672 & & & & 0,21 & 34,59 \\
2 & $-25,095$ & 4,858 & & & 0,96 & 7,94 \\
3 & 6,506 & 0,016 & & & 0,90 & 12,43 \\
4 & $-25,016$ & 4,862 & $-0,011$ & & & 0,96 & 8,17 \\
5 & $-1,445$ & 1,985 & & & 0,93 & 10,06 \\
6 & 1,890 & 0,022 & & & 0,80 & 17,31 \\
7 & 1,165 & 0,267 & & & & 0,90 & 12,57 \\
8 & $-31,539$ & 5,946 & $-0,024$ & $-0,002$ & & 0,95 & 8,32 \\
9 & $-29,005$ & 5,480 & $-0,002$ & & & 0,96 & 8,09 \\
10 & $-1,569$ & 1,953 & 0,085 & & & 0,93 & 10,30 \\
11 & 1,729 & 0,113 & & & & 0,88 & 13,31 \\
12 & 329,366 & $-125,944$ & 17,719 & $-1,043$ & 0,022 & 0,95 & 8,47 \\
13 & 11,399 & 0,011 & & & & 0,90 & 12,01 \\
\hline
\end{tabular}

$\beta_{\mathrm{n}}=$ coeficientes da regressão.

Equação 2

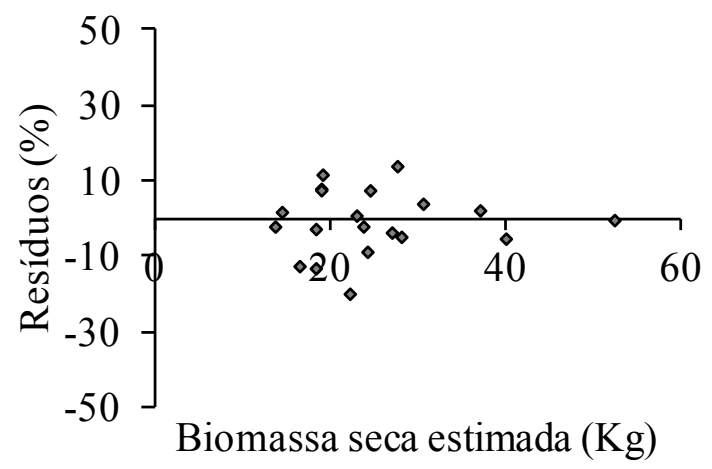

Equação 9

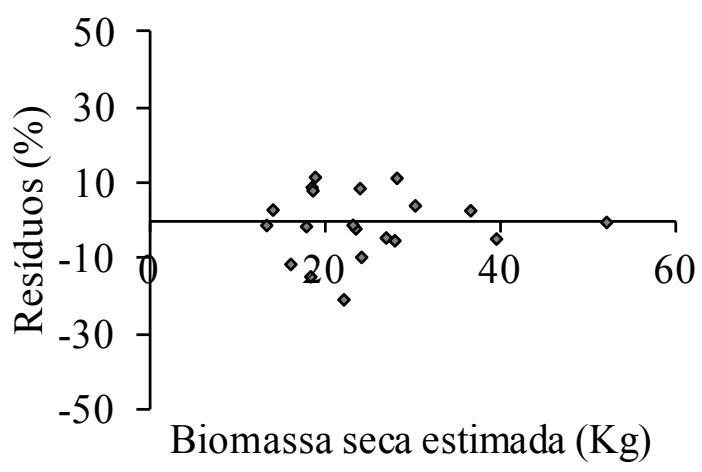

Equação 8

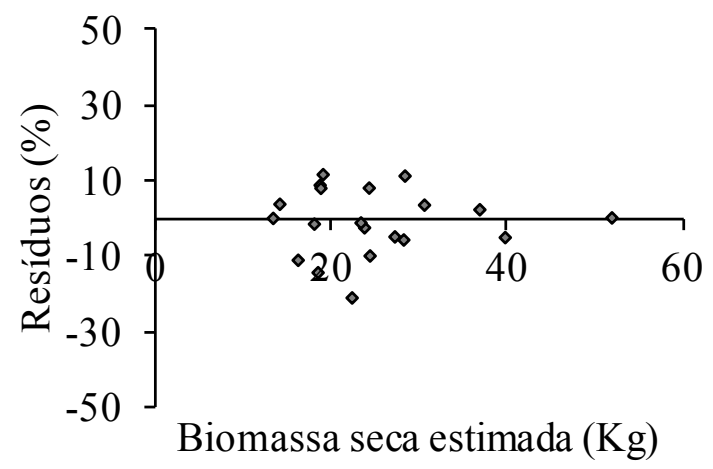

Equação 12

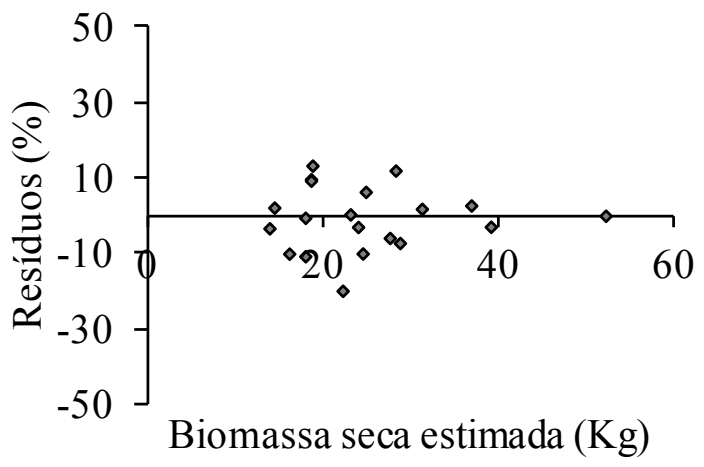

Figura 3. Distribuição dos resíduos dos modelos 2, 8, 9 e 12 para estimativa da biomassa total de T. cassinoides. 
Tabela 6. Condicionantes da regressão para os resíduos dos modelos de melhor ajuste para biomassa total de caixeta no Estado do Paraná.

\begin{tabular}{|c|c|c|c|c|}
\hline Numeração & Modelo & $\mathbf{W}$ & SW & DW \\
\hline Equação 2 & $\mathrm{Y}=\beta 0+\beta 1$ dap & $1,82^{\mathrm{ns}}$ & $0,98^{\mathrm{ns}}$ & $1,94^{\text {ns }}$ \\
\hline Equação 8 & $\mathrm{Y}=\beta 0+\beta 1 *$ dap $+\beta 2 * \mathrm{dap}^{2}+\beta 3\left(\mathrm{dap}^{2} \mathrm{~h}\right)$ & $9,32^{\mathrm{ns}}$ & $0,98^{\text {ns }}$ & $1,94^{\mathrm{ns}}$ \\
\hline Equação 9 & $\mathrm{Y}=\beta 0+\beta 1 *$ dap $+\beta 2\left(\mathrm{dap}^{2} \mathrm{~h}\right)$ & $10,73^{\text {ns }}$ & $0,94^{\mathrm{ns}}$ & $1,62^{*}$ \\
\hline Equação 12 & $\mathrm{Y}=\beta 0+\beta 1 *$ dap $+\beta 2 *$ dap $^{2}+\beta 3 *$ dap $^{3}+\beta 4 *$ dap $^{4}$ & $10,73^{\text {ns }}$ & $0,99^{\text {ns }}$ & $2,04^{\mathrm{ns}}$ \\
\hline
\end{tabular}

$*$ Significativo a $5 \%$ de probabilidade. $\mathrm{ns}=$ não significativo a $5 \%$ de probabilidade. $\mathrm{W}=$ White; $\mathrm{SW}=$ Shapiro-Wilk; DW $=$ Durbin-Watson; $\beta \mathrm{n}=$ coeficientes da regressão; dap $=$ diâmetro à altura do peito; $\mathrm{h}=$ altura total.

Tal qual encontrado nos ajustes propostos dos modelos, a alta correlação entre as variáveis dependentes com o dap também foi observada por Silveira (2009), inferindo que tanto em sua forma pura como combinada, a presença dessa variável é indispensável aos modelos que objetivam à estimativa de biomassa e carbono total. Conclusão semelhante foi mencionada por Giongo et al. (2011), em seu estudo analisando biomassa em um plantio de Pinus eliottii no Paraná, o que indica essa potencialidade para múltiplas espécies.

Para os modelos de estimativa do carbono total, as estatísticas de ajuste observadas indicaram o parâmetro $\mathrm{R}_{\text {aj }}^{2}$,variando entre 0,79 e 0,97 , demonstrando um bom desempenho no ajuste, com ressalva para o modelo 1 $\left(\mathrm{R}_{\text {aj. }}^{2}=0,17\right)$, notado por apresentar baixo desempenho também na estimativa de biomassa total. Ainda assim, os indicadores de ajustes dos demais modelos demonstraram alto grau de ajuste da variável dependente em função das variáveis independentes (Tabela 7).

Como ocorrido para avaliação da biomassa total, alguns modelos obtiveram comportamento similar, porém, aquele que melhor estimou o carbono total de acordo com os parâmetros $\mathrm{R}_{\text {aj }}^{2}(0,97)$ e $\mathrm{S}_{\mathrm{yx}}(7,91 \%)$ foi o número 8. Observou-se, entretanto, que outros modelos como o 12 e o 9 também se mostraram adequados para estimar o carbono total, apresentado valores de $\mathrm{R}_{\text {aj }}^{2}$ igual a 0,97 e 0,96 e $\mathrm{S}_{\mathrm{yx}}(\%)$ de 8,09 e 8,47 , respectivamente.

Os números encontrados no presente trabalho evidenciam a adequada capacidade para extrapolação dos resultados a partir dos referidos modelos, pois se insere no intervalo de valores aceitáveis pela literatura como capazes de predizer com relativa acurácia a biomassa e carbono (Miranda et al., 2012; Lacerda et al., 2009; Soares et al., 2002). A representação gráfica dos resíduos para o carbono total é apresentada na Figura 4.
Devido à baixa amplitude da análise residual, inferese que as estimativas são mais exatas, proporcionando uma variância homogênea. Souza et al. (2013), em análise da dispersão dos resíduos para carbono orgânico total de bracatingais no Paraná, obtiveram semelhante distribuição de erros para os modelos tradicionais testados, onde através das mesmas estatísticas de ajustes consideradas no presente trabalho, resultaram em modelos apropriados para potenciais estimativas.

Ao analisar as condicionantes da regressão (Tabela 8), observa-se que os resíduos das equações 8 e 9 não foram considerados normais. Embora as estatísticas de ajuste tenham apontado a possibilidade do uso desses modelos, neste caso, optou-se por considerar que o melhor modelo para estimativa do carbono total é a equação 12 , visto que a mesma foi a única que aceitou a hipótese de normalidade $\left(\mathrm{H}_{0}\right)$. Aliado a isso, essa equação tem como variável independente apenas o dap, de fácil obtenção e boa correlação, conforme já discutido anteriormente.

Nota-se, ainda quanto ao modelo 12, que o mesmo apresenta valores significativos para o teste de Durbin-Watson (independência), indicando que os resíduos apresentam algum tipo de dependência. Esta dependência, porém, não é um problema conforme Schumacher et al. (2013), segundo o qual, valores do teste Durbin-Watson menores que 1 e maiores que 3 são tidos como problemáticos, enquanto que 2 é o valor ideal. Para este modelo, o valor foi de 1,66, sendo considerado aceitável.

A definição da melhor equação com base na normalidade é apontada também por Ré (2011), onde menciona que quando os valores dos coeficientes de determinação ajustados são altos e os valores de erro padrão são próximos, a normalidade é um bom indicativo de ajuste. 
Tabela 7. Coeficientes e indicadores estatísticos dos modelos para carbono total de T. cassinoides.

\begin{tabular}{|c|c|c|c|c|c|c|c|}
\hline Modelos & $\beta_{0}$ & $\beta_{1}$ & $\beta_{2}$ & $\boldsymbol{\beta}_{3}$ & $\beta_{4}$ & $\mathbf{R}^{2}{ }_{\text {aj. }}$ & $S_{y x}(\%)$ \\
\hline 1 & $-6,776$ & 1,752 & & & & 0,17 & 39,54 \\
\hline 2 & $-14,223$ & 2,500 & & & & 0,95 & 9,33 \\
\hline 3 & 2,261 & 0,008 & & & & 0,86 & 16,46 \\
\hline 4 & $-12,905$ & 2,565 & $-0,191$ & & & 0,95 & 9,39 \\
\hline 5 & $-2,933$ & 2,285 & & & & 0,87 & 15,68 \\
\hline 6 & $-3,818$ & 0,887 & & & & 0,86 & 16,17 \\
\hline 7 & 0,087 & 0,305 & & & & 0,79 & 20,04 \\
\hline 8 & $-30,083$ & 5,243 & $-0,083$ & $-0,003$ & & 0,97 & 7,91 \\
\hline 9 & $-21,280$ & 3,622 & $-0,004$ & & & 0,96 & 8,47 \\
\hline 10 & $-2,722$ & 2,340 & $-0,145$ & & & 0,86 & 16,46 \\
\hline 11 & $-2,933$ & 0,762 & & & & 0,87 & 15,68 \\
\hline 12 & 284,558 & $-105,063$ & 14,140 & $-0,804$ & 0,017 & 0,97 & 8,09 \\
\hline 13 & 4,693 & 0,006 & & & & 0,86 & 16,08 \\
\hline
\end{tabular}

$\beta_{\mathrm{n}}=$ coeficientes da regressão; $\mathrm{R}_{\mathrm{aj}}^{2}=$ coeficiente de determinação ajustado; $\mathrm{S}_{\mathrm{yx}} \%=$ erro padrão da estimativa.

Equação 8

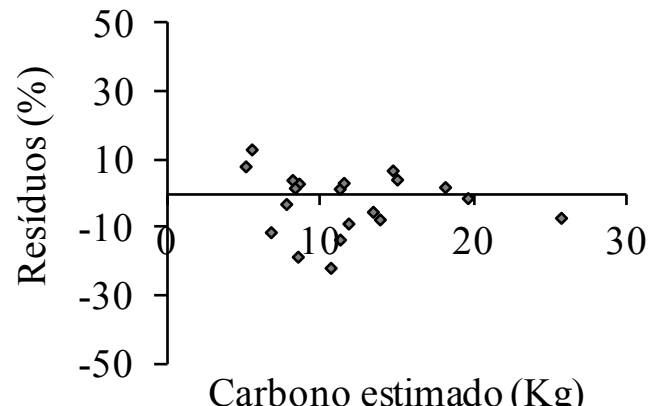

Equação 9

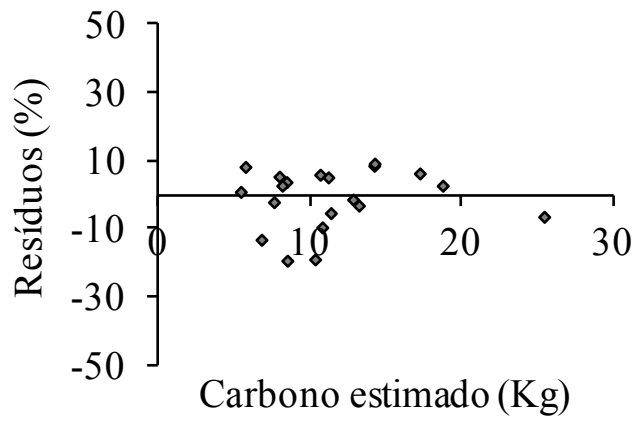

Equação 12

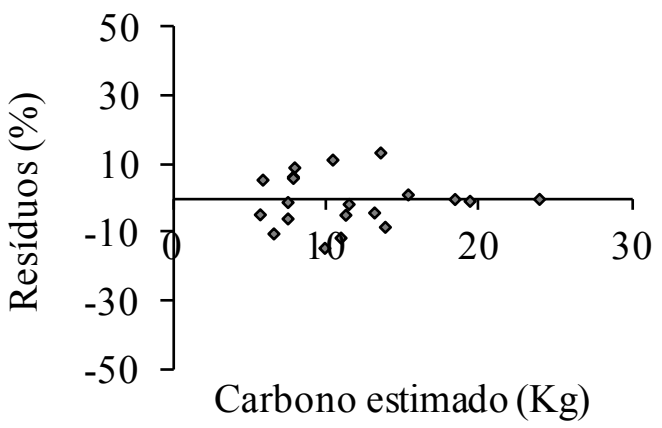

Figura 4. Análise residual dos modelos 8, 9 e 12 para estimativa do carbono total de T. cassinoides. 
Tabela 8. Condicionantes da regressão para os resíduos dos modelos de melhor ajuste para carbono total de caixeta no Estado do Paraná.

\begin{tabular}{|c|c|c|c|c|}
\hline Numeração & Modelo & $\mathbf{W}$ & SW & DW \\
\hline Equação 8 & $\mathrm{Y}=\beta 0+\beta 1 *$ dap $+\beta 2 * \operatorname{dap}^{2}+\beta 3\left(\operatorname{dap}^{2} \mathrm{~h}\right)$ & $0,96^{\mathrm{ns}}$ & $14,58^{*}$ & $2,14^{\mathrm{ns}}$ \\
\hline Equação 9 & $\mathrm{Y}=\beta 0+\beta 1 *$ dap $+\beta 2\left(\right.$ dap $\left.^{2} \mathrm{~h}\right)$ & $0,98^{\mathrm{ns}}$ & $17,58^{*}$ & $2,18^{\text {ns }}$ \\
\hline Equação 12 & $\mathrm{Y}=\beta 0+\beta 1 *$ dap $+\beta 2 *$ dap $^{2}+\beta 3 *$ dap $^{3}+\beta 4 *$ dap $^{4}$ & $11,19^{\text {ns }}$ & $0,97^{\mathrm{ns}}$ & $1,66^{*}$ \\
\hline
\end{tabular}

$*=$ Significativo a $5 \%$ de probabilidade. $\mathrm{ns}=$ não significativo a $5 \%$ de probabilidade. $\mathrm{W}=$ White; $\mathrm{SW}=$ Shapiro-Wilk; $\mathrm{DW}=$ Durbin-Watson; $\beta \mathrm{n}=$ coeficientes da regressão; dap = diâmetro à altura do peito; $\mathrm{h}=$ altura total.

\section{Conclusões}

Os modelos ajustados para estimar biomassa seca total e carbono total de T. cassinoides mostraram-se eficientes, considerando os indicadores de ajustes observados.

Para a biomassa total, o modelo $2(\mathrm{Y}=\beta 0+\beta 1 *$ dap $)$ mostrou-se superior aos demais, e para o carbono total, o melhor ajuste foi dado pelo modelo $12(\mathrm{Y}=\beta 0+\beta 1 *$ dap $+\beta 2 *$ dap $^{2}+\beta 3 *$ dap $^{3}+\beta 4 *$ dap $\left.^{4}\right)$, ambos apresentando bons valores de $\mathrm{R}_{\text {aj }}^{2}$ e $\mathrm{S}_{\mathrm{yx}}(\%)$ e baixa variação na análise gráfica dos resíduos.

A variável altura total, utilizada na forma isolada, revelou-se inadequada para explicar as variáveis biomassa total e carbono total em função da baixa correlação com as variáveis independentes avaliadas.

\section{Referências}

BATISTA, J. L. F.; MARQUESINI, M.; VIANA, V. M. Equações de volume para árvores de caxeta (Tabebuia cassinoides) no Estado de São Paulo e sul do Estado do Rio de Janeiro. Scientia Forestalis, Piracicaba, v. 65, p. 162-175, 2004.

GALVÃO, F.; RODERJAN, C. V.; KUNIYOSHI, Y. S.; ZILLER, S. R. Composição florística e fitossociológica de caixetais do litoral do Estado do Paraná - Brasil. Floresta, Curitiba, v. 32, n. 1, p. $17-$ 39, 2002.

GIONGO, M.; SILVA, D. B.; KOEHLER, H. S.; MARCHETTI, M. Inventário de biomassa em um plantio de Pinus eliottii Engelm. aos 23 anos de idade. Journal of Biotechnology and Biodiversity, Tocantins, v. 2, n. 3, p. 81-86, 2011.

KOEHLER, H. S.; WATZLAWICK, L. F.; KIRCHNER, F. F.; VALÉRIO, A. F. Fontes de erros nas estimativas de biomassa e carbono fixado na Floresta Ombrófila Mista. Biomassa e Energia, Viçosa, MG, v. 2, n. 1, p. 69-77, 2005.

LACERDA, J. S.; COUTO, H. T. Z.; HIROTA, M. M.; PASISHNYK, N.; POLIZEL, J. L. Estimativa de biomassa e carbono em áreas restauradas com plantio de essências nativas. METRVM, São Paulo, n. 5, 2009.

MARTINS, P. J. Biomassa vegetal, estoque de carbono e dinâmica em um fragmento de Floresta Ombrófila Mista Montana. 2011. 86 f. Dissertação (Mestrado em Agronomia) - Universidade Estadual do Centro-Oeste, Guarapuava.
MIRANDA, D. L. C. de; MELO, A. C. G. de; SANQUETTA, C. R. Equações alométricas para estimativa de biomassa e carbono em árvores de reflorestamentos de restauração. Revista Árvore, Viçosa, MG, v. 35, n. 3, Edição Especial, p. 679-689, 2011.

MIRANDA, D. L. C.; SANQUETTA, C. R.; COSTA, L. G. S.; CORTE, A. P. D. Biomassa e carbono em Euterpe oleracea Mart. na ilha do Marajó - PA. Floresta e Ambiente, Rio de Janeiro, v. 19, n. 3, p. 336-343, 2012.

MOGNON, F.; DALLAGNOL, F. S.; SANQUETTA, C. R.; CORTE, A. P. D.; BARRETO, T. G. Uma década de dinâmica de fixação de carbono na biomassa arbórea em Floresta Ombrófila Mista no Sul do Paraná. Floresta, Curitiba, v. 43, n. 1, p. 153-164, 2013.

POPPE, M. K. (Coord.). Manual de capacitação sobre mudanças do clima e projetos de mecanismos de desenvolvimento limpo (MDL). Brasília, DF: Centro de Gestão de Estudos Estratégicos, 2008. $276 \mathrm{p}$.

RÉ, D. S. Equações volumétricas e produção de madeira em plantios mistos visando à Restauração da Floresta Estacional Semidecidual. 2011, 57 f. Dissertação (Mestrado em Ciências Florestais) - Faculdade de Ciências Agronômicas da UNESP, Botucatu.

RIBEIRO, S. C.; JACOVINE, L. A. G.; SOARES, C. P. B.; MARTINS, S. V; NARDELLI, A. M. B; SOUZA, A. L. Quantificação de biomassa e estimativa de estoque de carbono em uma capoeira da zona da mata mineira. Revista Árvore, Viçosa, $\mathrm{MG}$, v. 34, n. 3 , p. 495-504, 2010.

SANQUETTA, C. R; CORTE, A. P. D.; BALBINOT, R.; ZILLIOTTO, M. A. B. Proposta metodológica para quantificação e monitoramento do carbono estocado em florestas plantadas. In: SANQUETTA, C. R.; ZILliOTTO, M. A. (Org.). Mercado de carbono: mercado e ciência. Curitiba: Imprensa Universitária da UFPR, 2004. v. 1. p.120-150.

SCHNEIDER, P. R.; FINGER, C. A. G.; HOPPE, J. M.; GIACOMELLI SOBRINHO, V.; SCHNEIDER, P. S. P. Método de derivação do volume em biomassa e carbono: uma aplicação em Platanus $x$ acerifolia (Aiton) Wild. In: SIMPÓSIO LATINOAMERICANO SOBRE MANEJO FLORESTAL, 3., 2004, Santa Maria, RS. Anais... Santa Maria, RS: UFSM, 2004. p. 381-388.

SCHNEIDER, P. R.; SCHNEIDER, P. S. P.; SOUZA, C. A. M. Análise de regressão aplicada à Engenharia Florestal. 2. ed. Santa Maria, RS: UFSM, Centro de Pesquisas Florestais, 2009. 294 p.

SCHUMACHER, M. V.; WITSCHORECK, R.; CALIL, F. N.; LOPES, V. G. Biomassa e nutrientes no corte raso de um povoamento de Pinus taeda L. de 27 anos de idade em Cambará Do Sul - RS. Ciência Florestal, Santa Maria, RS, v. 23, n. 2, p. 321-332, 2013. 
SILVEIRA, P.; KOEHLER, H. S.; SANQUETTA, C. S.; ARCE, J. E. $O$ estado da arte na estimativa de biomassa e carbono em formações florestais. Floresta, Curitiba, v. 38, n. 1, p. 185-206, 2008.

SILVEIRA, P. Ajuste de modelos matemáticos para estimar biomassa aérea em Floresta Ombrófila Densa. Floresta, Curitiba, v. 39, n. 4, p. 743-752, 2009.

SILVEIRA, P. Estimativa da biomassa e carbono acima do solo em um fragmento de Floresta Ombrófila Densa utilizando o método da derivação do volume comercial. Floresta, Curitiba, v. 40, n. 4, p. 789-800, 2010.
SOARES, C. P. B.; OLIVEIRA, M. L. R. Equações para estimar a quantidade de carbono na parte aérea de árvores de eucalipto em Viçosa, Minas Gerais. Revista Árvore, Viçosa, MG, v. 26, n. 5, p. 553-539, 2002.

SOUZA, R. F.; MACHADO, S. A.; FIGUEIREDO FILHO, A.; KOEHLER, H. S. Modelagem do carbono orgânico total e da lenha por unidade de área para bracatingais nativos. Ciência Florestal, Santa Maria, RS, v. 23, n. 1, p. 117-127, 2013. 
